\title{
REFLEXIÓN PEDAGÓGICA EN BASE A CASOS Y DOMINIO DE LENGUAJE ACADÉMICO EN ESTUDIANTES DE CUARTO AÑO DE PEDAGOGÍA EN EDUCACIÓN BÁSICA ${ }^{1}$
}

\author{
Soledad Concha, ${ }^{2}$ Carolina Hernández ${ }^{3}$, Francisca del \\ Río $^{4}$, Francisca Romo ${ }^{5}$, Lorena Andrade ${ }^{6}$
}

\begin{abstract}
RESUMEN
Se investigó la capacidad de los estudiantes de Pedagogía en Educación Básica de Santiago para reflexionar pedagógicamente en base a un caso (RPEC) y la relación entre dicha capacidad y el dominio de lenguaje académico. Los participantes observaron un video de una clase de Lenguaje y Comunicación y reflexionaron por escrito respecto de los sustentos teóricos de las decisiones pedagógicas de la profesora, su enseñanza y de las posibles vías de mejoramiento.

Los puntajes promedio obtenidos muestran bajos desempeños entre los participantes, quienes no revelaron dominio de la teoría que subyace a la clase observada y recurrieron a explicaciones generales, no técnicas. Los resultados muestran una correlación entre el desempeño en RPEC y la capacidad para organizar un texto sobre la base de conceptos teóricos incluidos en la reflexión escrita solicitada. Esta relación y los resultados obtenidos son analizados en el marco de la formación inicial docente.

Palabras clave: Reflexión pedagógica, lenguaje académico, formación inicial docente.
\end{abstract}

\section{PEDAGOGIC REFLECTION BASED ON CASES AND COMMAND OF ACADEMIC TERMS IN STUDENTS TAKING THE FOURTH YEAR OF ELEMENTARY TEACHING FORMATION}

\section{ABSTRACT}

This study explored the ability of Chilean students of Elementary teaching to produce written case-based teacher reflections (CBTR), and the relationship between that ability and their command of academic terms. Participants watched a video of a Spanish language lesson and reflected about the theoretical basis of the teacher's pedagogical decisions, her teaching method and ways to improve it.

Average outcomes reveal that participants lack domain of the theory behind the observed class and provided common sense, not technical responses. A correlation was found between results in CBTR and participants' ability to organize a text based on the theoretical concepts involved in the reflection. The results are analyzed within the broader context of initial teaching formation.

Keywords: Teacher reflection, academic language, initial teaching education

1 Este trabajo fue financiado con fondos de investigación del Consejo Nacional de Educación del año 2012. El equipo de investigadoras agradece los aportes a este trabajo ofrecidos por: Jeanne R. Paratore, de la Universidad de Boston, EE.UU.; Paola Uccelli, de la Universidad de Harvard, EE.UU. y Silvia Romero, de la Universidad Autónoma de San Luis Potosí, México.

2 Doctora en Educación, académica de la Facultad de Educación de la Universidad Diego Portales, Santiago, Chile. Contacto: soledad.concha@mail.udp.cl

3 Magíster en Lingüística, académica de la Facultad de Educación de la Universidad Diego Portales, Santiago, Chile.

4 Doctora en Psicología, académica de la Facultad de Educación de la Universidad Diego Portales, Santiago, Chile.

5 Socióloga, asistente de investigación en CPCE, Universidad Diego Portales, Santiago, Chile.

6 Licenciada en Educación y profesora de Lenguaje y Comunicación, profesora de la Unidad de Alfabetización Académica, Universidad Diego Portales, Santiago, Chile. 


\section{REFLEXIÓN PEDAGÓGICA EN BASE A CASOS \\ Y DOMINIO DE LENGUAJE ACADÉMICO \\ EN ESTUDIANTES DE CUARTO AÑO DE PEDAGOGÍA EN EDUCACIÓN BÁSICA}

\section{Introducción}

La reflexión pedagógica ha recibido gran atención en la literatura durante décadas, por su asociación con la experticia en la enseñanza. Los estudios, sin embargo, son diversos respecto de la manera en que conceptualizan esta noción, lo que pone en cuestionamiento su valor, tanto como la posibilidad de identificar métodos sistemáticos para desarrollar este tipo particular de razonamiento entre los futuros profesores.

Un cuerpo reducido de estudios ha explorado la idea de que la escritura es un buen medio para desarrollar la reflexión pedagógica en programas de pedagogía. Esta propuesta se basa en la noción de que escribir no es meramente un medio de comunicación, sino también una oportunidad para construir y transformar nuestros conocimientos.

El siguiente trabajo se funda particularmente en la evidencia recogida en estudios acerca de la cognición de los profesores novatos y la de los expertos, según los cuales una de las características diferenciadoras clave de ambos grupos es la capacidad de analizar una sala de clases en función de conocimientos especializados y propios de la profesión docente. Así también, el estudio se basa en trabajos que proponen que la escritura es un medio privilegiado para evaluar y para enseñar a los estudiantes a reflexionar pedagógicamente durante su formación.

En este estudio se entiende la reflexión pedagógica en base a casos como la capacidad para analizar por escrito los fundamentos teóricos de las decisiones pedagógicas observadas en una clase dictada por otro profesor, usando para ello -de manera articuladalos distintos saberes relevantes de la pedagogía: disciplinarios, de 
didáctica general, de didáctica específica, del desarrollo de los niños y de los contextos de la enseñanza.

Hay evidencia disponible en Chile respecto de las dificultades que presentarían los universitarios en general y los estudiantes de pedagogía en particular, para comunicarse por escrito en sus programas. Así, una propuesta de evaluación y de enseñanza de la reflexión pedagógica escrita debe hacerse cargo de explorar la posible interacción entre los desempeños en esta habilidad y la capacidad de los estudiantes para producir textos académicos. En este trabajo esa exploración está guiada por la noción de lenguaje académico.

\section{Marco teórico}

Se ofrece en este apartado una revisión de algunos trabajos teóricos y empíricos que abordan la noción de reflexión pedagógica en profesores expertos y novatos, así como de aquellos que investigan su representación y evaluación en distintas formas escritas. Esta revisión incluye asimismo una descripción de la noción de lenguaje académico, para dar sustento teórico a la exploración de una relación entre la capacidad para reflexionar pedagógicamente por escrito y el dominio de recursos sofisticados de la lengua y del discurso.

\subsection{La reflexión pedagógica en base a casos}

Una revisión de la teoría disponible respecto de la reflexión pedagógica revela que se trata de una preocupación central en el área de la educación. Los estudios que exploran la idea de que los futuros profesores deben aprender a pensar reflexivamente acerca de la enseñanza se basan en distintas conceptualizaciones: reflexión crítica pedagógica (Harrington, Quinn-Leeringy Hodson, 1996), reflexión práctica (Luk, 2008), habilidad reflexiva (Braun y Cumpler, 2004), pensamiento pedagógico reflexivo (Lee, 2005), razonamiento pedagógico (Youngs y Bird, 2010; Herman, 1998) y enseñanza reflexiva (Silcock, 1994). Esta heterogeneidad teórica ha sido discutida por distintos autores, quienes sostienen que, a pesar de la relevancia de su objeto de estudio, el concepto se ha vuelto un tanto confuso en la literatura disponible (Mclellan, 2008; Silcock, 1994; Lee, 2005; Braun y Crumpler, 2004). 
La mayoría de los artículos relacionados con la reflexión pedagógica hacen referencia al razonamiento pedagógico de Shulman (1987) y a las reflexiones en la práctica y acerca de la práctica de Schön (1983). Los dos autores estudian la noción de reflexión en relación con la práctica profesional y comparten la idea de que los profesores pueden pensar en sus decisiones instruccionales, lo que a su vez les permite mejorar su práctica. Para sustentar la toma de decisión y el desarrollo profesional, los profesores deben apoyarse sobre una base sólida de conocimiento (Darling Hammond y Bransford, 2005; Berliner, 1986; Ben-Peretz, 2011). La propuesta es que los eventos del aula pueden ser entendidos y analizados por referencia a distintas fuentes de conocimiento especializado, las que deberían estar disponibles para la reflexión.

Los profesores novatos no serían tan capaces de reflexionar en la acción, como sí lo son los expertos. Esto se debería a una base precaria de conocimiento, a la falta de habilidades metacognitivas, o bien, al hecho de que las complejidades de la enseñanza no dejarían espacio de atención disponible para la reflexión (Darling Hammond y Bransford, 2005; Berliner, 1986; Schemp y Woorons, 2006; Carter y Richardson, 1989; Davis, 2006; Borko y Livingston, 1989). Berliner (1986) encontró siete rasgos que caracterizan a los profesores expertos y que los distinguen con claridad de los novatos: infieren sobre la base a su observación de eventos y objetos del aula, categorizan los problemas (categorización de orden superior), rápidamente identifican patrones de conducta, se demoran más en examinar los problemas del aula, son sensibles a los cambios en el contexto de la enseñanza, son flexibles (planean sobre la marcha, lo que se opone a la fijación funcional de los novatos) y autorregulan su conducta.

No obstante la capacidad limitada de realizar este tipo de razonamiento durante la enseñanza, los autores revisados coinciden en que los profesores novatos deberían ser capaces de reflexionar en torno a eventos del aula, como parte de su proceso de convertirse en profesores expertos (Berliner, 1986; Darling Hammond y Bransford, 2005; Carter y Richardson, 1989; Schemp y Woorons, 2006; Davis, 2006). Se trataría de la capacidad de analizar situaciones de aula sobre la base de distintos saberes, un ejercicio que exige revisar de qué 
manera se presentan estos saberes a los futuros profesores durante su formación, de modo que estén disponibles para este tipo de análisis.

Siguiendo esta idea, Carter y Richardson (1989) proponen un método de enseñanza para la formación inicial docente que denominan «conocimiento estructurado en torno a eventos». Este método, muy similar a la propuesta de «conocimiento situado basado en casos» de Kim y Hannafin (2008), propone que se les presenten a los profesores en formación articuladamente distintas fuentes de conocimiento relacionadas con eventos del aula. Los estudiantes de pedagogía serían luego enfrentados a casos de aula que deben analizar desde distintas perspectivas (manejo de aula, tipos de preguntas, organización del espacio, evaluación, entre otros), considerando no solo qué y cómo se enseña en una sala en particular, sino también las explicaciones que sustentan las decisiones de un profesor y qué se podría cambiar para mejorar la enseñanza.

\subsection{Alfabetización pedagógica}

Mclellan (2008) introduce el concepto de «alfabetización pedagógica» para referirse a la capacidad de comprender y producir un discurso al interior de la comunidad académica de la educación. Su descripción del concepto se basa en los principios de la escritura como medio para aprender (Emig, 1977) y considera la alfabetización como una herramienta cognitiva que puede promover el desarrollo del conocimiento pedagógico. De acuerdo con la autora, para aprender cómo enseñar, un estudiante de pedagogía debe atravesar un proceso que incluye leer y escribir. Un profesor que ha tenido la oportunidad de leer acerca de la teoría y la práctica de la enseñanza y el aprendizaje puede acceder al conocimiento contenido en esos textos, para razonar con ellos en su práctica. Sin la representación de ese conocimiento, la enseñanza se limita a la ejecución automática y los profesores no pueden ser flexibles (adaptarse a lo inesperado), o bien, mejorar sus prácticas.

Escribir durante la formación de profesores es visto por la autora como una oportunidad de construir el conocimiento pedagógico discursivamente. Por ejemplo, en relación con el conocimiento pedagógico del contenido de Shulman (1987), esa manera única 
de pensar que permite a los profesores transformar el conocimiento disciplinario en formas que el aprendiz puede comprender (Mclellan, 2008, p. 1988), la autora declara que se puede representar tanto simbólicamente como en acciones pedagógicas. Si se representa simbólicamente a través de la escritura, el conocimiento pedagógico del contenido puede ser transformado por los futuros profesores, a la vez que podrá ser observado por sus instructores. Al representar su conocimiento teórico y práctico por escrito, los futuros profesores tienen la oportunidad de hacer de este conocimiento un objeto de reflexión: pueden manipularlo, integrarlo, reestructurarlo, controlarlo y transformarlo.

Una cuestión relevante asociada a la noción de alfabetización pedagógica es la posibilidad de identificar géneros discursivos específicos que constituyan formas convencionales de construir, representar y comunicar el conocimiento pedagógico en la comunidad académica de la educación. Si bien no se extiende a otros géneros específicos de la formación inicial docente, la autora refiere el trabajo de Ohlsson (1995 en Mclellan, 2008) según el cual géneros como el ensayo académico funcionarían como ejercicios epistémicos que permiten a los futuros profesores organizar y clasificar sus conocimientos en estructuras que visibilizan relaciones entre distintos conceptos. El ejercicio de producción de ensayos contribuiría así tanto a la representación estructurada, como a la revisión y reformulación de su conocimiento experto.

\subsection{Reflexión pedagógica y escritura: los géneros escritos de la formación inicial docente}

Algunos estudios disponibles han explorado el uso de la escritura como medio para desarrollar hábitos de una reflexión pedagógica basada en fuentes relevantes de conocimiento (Davis, 2006; BarksdaleLadd et al, Draper, King, Oropallo y Radencich, 2001; Le Fevre, 2011; Braun y Cumpler, 2003; Lee, 2005; Luk, 2008). Como explica Davis (2006), distintas tareas de escritura que se utilizan en la formación inicial docente tienen el potencial de promover la integración de diversos conocimientos y la reflexión acerca de ellos. Entre otras, el diálogo, la investigación-acción, la investigación realizada por 
profesores, la escritura reflexiva y el análisis de casos. Todas estas tareas involucran la reflexión articulada de aspectos clave sobre los aprendices y el aprendizaje, respecto del conocimiento disciplinario, la evaluación y la enseñanza y los contextos de la enseñanza.

En su propio trabajo con diarios reflexivos en la formación inicial docente, Davis (2006) sugiere que el lenguaje es un mediador en el proceso de aprendizaje de lo que ella llama «reflexión productiva». En este tipo de escritura, los estudiantes entregan evidencias para secundar sus afirmaciones, hacen propuestas alternativas, cuestionan supuestos, identifican los resultados de la enseñanza, evalúan y juzgan la enseñanza. Por su parte, la reflexión improductiva se caracteriza por una escritura centrada en la descripción de situaciones de enseñanza, listas de ideas sin relación y una perspectiva crítica y de juicios sin fundamento empírico.

Se puede encontrar también otro cuerpo de investigaciones que exploran tareas escritas como medios para desarrollar la reflexión pedagógica, pero que conceptualizan la reflexión de forma diferente; por ejemplo, como una oportunidad para relacionar el ámbito personal con el profesional (Barksdale-Ladd et al, 2001; Braun y Cumpler, 2004; Le Fevre, 2011), como cuando se recuperan experiencias previas de aprendizaje, de modo que los futuros profesores recuerden cómo era ser estudiantes y qué estrategias de enseñanza les ayudaban más. Las memorias sociales, las narraciones de niños, las autobiografías, entre otros géneros más narrativos, se presentan en la literatura especializada en relación con la noción de que el conocimiento pedagógico se puede representar narrativamente, lo que afecta la percepción y las decisiones de los docentes (Le Fevre, 2011). En este marco, se promueve que los estudiantes de pedagogía escriban para que den voz y reflexionen en torno a sus teorías implícitas, sus actitudes y su conocimiento respecto de la enseñanza.

\subsection{La evaluación de la reflexión pedagógica escrita}

Hay disponibles pocos estudios que reporten la construcción de instrumentos válidos y confiables para evaluar la reflexión pedagógica (Youngs y Birds, 2010; Fund, Court y Kramarski, 2002). Es posible encontrar, también, algunos trabajos que dan cuenta de estudios 
de casos de reflexión pedagógica (Luk, 2008; Lee, 2005; Davis, 2006), junto con detalles de los procedimientos de análisis. No obstante escasas, es interesante la coincidencia que existe entre estas investigaciones.

En primer lugar, respecto de la funcionalidad que atribuyen al ejercicio de evaluar la reflexión pedagógica por escrito, todos ellos sostienen que la escritura es un medio privilegiado para evaluar y para enseñar a los futuros profesores a reflexionar pedagógicamente durante su formación. Hay incluso evidencia de que la aplicación sistemática de ejercicios de reflexión pedagógica escrita logra, gradualmente, mejorar las capacidades de los estudiantes en esta tarea (Fund et al., 2002). Lo anterior incluye retroalimentaciones recursivas a los estudiantes respecto de su nivel de logro, basadas en un modelo y criterios de evaluación determinados.

En segundo lugar, los autores coinciden en la amplitud y vaguedad del concepto de reflexión pedagógica y de la necesidad de modelos y procedimientos para sistematizarlo, de manera que sea posible transferirlo a los estudiantes. Se argumenta respecto de la importancia de familiarizar a los estudiantes no solo con los criterios de evaluación, sino que también con las características discursivas de la reflexión pedagógica, para promover una comprensión más profunda de lo que constituye este ejercicio y de cómo se construye con palabras (Luk, 2008).

En tercer lugar, llama la atención que la totalidad de los autores gradúa la calidad de la reflexión pedagógica en polos que van desde la mera descripción de lo observado o realizado y los juicios sin fundamentos, hasta una reflexión profunda o crítica que involucra afirmaciones con fundamentos, distintas perspectivas de análisis y articulación entre distintos conceptos. Dos elementos diferenciales de los trabajos referidos merecen ser resaltados. Por una parte, Youngs y Birds (2010) agregan a estos criterios de evaluación la necesidad de que los estudiantes demuestren un sólido dominio de los conceptos teóricos que utilizan para realizar su análisis. En esta lógica se considera de bajo nivel no solo que se cometan errores conceptuales, sino también que se utilicen los conceptos de manera 
vaga o imprecisa, o bien, que se nombren solamente en lugar de usarlos para realizar el análisis. Por otra parte, Fund et al. (2002) relevan la capacidad de los estudiantes para situarse, en el más alto nivel de la rúbrica, en un marco conceptual más amplio, ofreciendo generalizaciones conceptuales. Esta propuesta es similar a otras que describen el dominio teórico de los docentes como un conocimiento del campo en el que se insertan los conceptos y sus interrelaciones (Mclellan, 2008; Darling Hammond y Bransford, 2005).

Si bien hay diferencias en la manera en que los distintos autores abordan el problema de la escritura en relación con la reflexión pedagógica escrita, hay también coincidencias en sus propuestas acerca de la necesidad de un uso sofisticado de la lengua para realizar este ejercicio. Luk (2008) y Davis (2006) optan por realizar análisis discursivos de las muestras recogidas, con el fin de derivar características constantes en los trabajos de mayor nivel. De estos estudios es posible concluir que el ejercicio reflexivo requiere de un dominio sofisticado de recursos de la lengua, que permita a los estudiantes establecer relaciones entre las ideas presentadas, como los conectores, las relaciones lógicas y los marcadores discursivos. De hecho, Luk (2008) sostiene que el dominio discursivo es una variable que necesariamente se entrecruza con el desempeño de los estudiantes, lo que justifica la necesidad de una enseñanza explícita. Recurriendo al trabajo de Bereiter y Scardamalia (1987), Fund et al. (2002) incluyen en su modelo de evaluación la noción de que en los niveles más altos de reflexión pedagógica, los estudiantes deben poder coordinar una mayor cantidad de ideas (conocimientos teóricos, experiencia y creencias) y transformarlas en su conjunto para construir conocimiento nuevo por escrito. Este plano «formal», según proponen, es el que se gradúa a lo largo de la rúbrica, de modo que el nivel más alto se alcanza no solo incluyendo información acerca de qué se enseña y cómo, sino que siendo capaz de reflexionar coordinadamente con todos los conceptos relevantes para ese análisis.

Por último, es importante destacar las propuestas de Youngs y Birds (2010) y Fund et al. (2002) respecto de que es posible aspirar a la construcción de instrumentos para ser usados al interior de la formación inicial docente (embedded assessments) con niveles de 
confiabilidad y validez adecuados. Según proponen Youngs y Birds (2010), es de la mayor importancia que los formadores de profesores trabajen colectivamente en la aplicación de estos instrumentos y que estos puedan coincidir con sus creencias y concepciones respecto de la educación.

\subsection{Lenguaje académico}

Siguiendo el trabajo de Snow y Ucelli (2008), entendemos el lenguaje académico como un registro de la lengua que es común en la cultura del mundo académico. En términos de sus características, se trata de un dominio léxico, gramatical, discursivo y disciplinario que permite «empaquetar» ideas complejas y abstractas al escribir, tanto como comprender los textos de la escuela y de las comunidades académicas. Este registro escrito se utiliza en diversas comunidades académicas, de tal manera que es posible distinguir tanto particularidades lingüísticas en cada una de ellas, como elementos comunes.

El concepto de lenguaje académico, desde una perspectiva pragmática, asocia indisolublemente el lenguaje escrito con el contexto sociocultural en el que se produce. Quienes producen géneros escritos académicos al interior de comunidades particulares, no solo poseen dominio lingüístico, sino también saberes disciplinarios, de modo que logran construir discursivamente el conocimiento de su comunidad. Inspirados en el trabajo de Halliday y Matthiesen (2004 en Schleppegrel y Oliveira, 2006), estudios como el de Schleppegrel y Oliveira (2006) asumen que las formas lingüísticas son inseparables de los contenidos. En otras palabras, los escritores expertos de una disciplina particular construyen sus teorías, argumentos y opiniones en forma discursiva en un texto, usando los recursos del lenguaje académico.

Snow y Uccelli (2008) clasifican las características del lenguaje académico en cinco categorías: densidad informativa, organización de la información, selección léxica y uso de la gramática para representar la realidad. Todas estas categorías refieren a dominios que requieren cuatro tipos de conocimiento para su realización experta: lingüístico, de géneros discursivos, de estrategias de razonamiento y disciplinario. 
Por ejemplo, respecto de la organización de ideas a un nivel global, los textos escritos en este registro presentan evidencia de que se ha planificado la entrega de información, pues esta se presenta en un orden explícito (Ucelli, Dobbs y Scott en prensa) y se introduce y cierra con segmentos que la abordan de manera general. Una estructura tal demuestra no solo que se han considerado las necesidades informativas del lector y que se dominan los recursos discursivos para organizar un texto, sino también que el escritor sabe cómo organizar su conocimiento, en tanto conceptos teóricos y sus interrelaciones (Maclellan, 2008). Esta organización suele estar acompañada de marcadores metadiscursivos («en primer lugar», «respecto de lo anterior», etc.) u otras formas lingüísticas que encabezan secciones del texto, reflejando la función que cumplen estas últimas en un esquema de organización mayor.

Así también, y en relación con el dominio disciplinario, es característico del lenguaje académico el uso de conceptos abstractos o procesos como sujetos oracionales (metáforas gramaticales). Un escritor que domina una disciplina particular pone en posición de sujeto gramatical una noción abstracta como la migración campociudad, la didáctica de la lengua, el manejo del aula, para poder predicar respecto de ellas. Lo hace también cuando recoge en categorías abstractas una serie de elementos ya mencionados («este fenómeno recién descrito», «este proceso, esta jerarquía»), reflejando su comprensión profunda del conocimiento respecto del cual escribe (Schleppegrel y Oliveira, 2006; Snow y Ucelli, 2008), tanto como su dominio de la referencialidad lingüística.

Estas, entre otras convenciones que caracterizan al lenguaje académico, resultan ser selecciones que un escritor realiza cuando adecua su participación a contextos comunicativos reales, dentro de los cuales reconoce determinadas convenciones que son valoradas por una comunidad, tanto como medios para construir y acceder al conocimiento experto de dicha comunidad. Estas convenciones son clave para la participación en las comunidades discursivas (Carlino, 2007; Tapia, Burdiles y Arancibia, 2003). 
92 REFLEXIÓN PEDAGÓGICA EN BASE A CASOS Y DOMINIO DE LENGUAJE ACADÉMICO EN ESTUDIANTES DE CUARTO AÑO DE PEDAGOGÍA EN EDUCACIÓN BÁSICA - S. Concha,

C. Hernández, F. del Río, F. Romo, L. Andrade

\section{Metodología}

\subsection{Preguntas de investigación}

Cuatro preguntas guían esta investigación:

a) ¿Qué capacidad tienen los estudiantes de cuarto año de Pedagogía en Educación Básica para reflexionar pedagógicamente en base a casos, en modalidad escrita?

b) ¿Qué dominio de lenguaje académico demuestran los estudiantes de cuarto año de Pedagogía en Educación Básica, al producir reflexiones pedagógicas a partir de casos en modalidad escrita?

c) ¿Hay alguna relación entre la capacidad para reflexionar pedagógicamente basándose en casos, en modalidad escrita, y el dominio de lenguaje académico en los estudiantes de cuarto año de Pedagogía en Educación Básica?

d) ¿Hay diferencias en la capacidad de reflexionar pedagógicamente sobre la base de casos y en el dominio de lenguaje académico entre los estudiantes de cuarto año de Pedagogía en Educación Básica de instituciones con promedios en la prueba Inicia, alto, medio y bajo?

\subsection{Procedimientos de selección de la muestra}

La muestra estuvo constituida por 82 estudiantes de cuarto año de la carrera de Pedagogía en Educación General Básica. Con el objeto de observar el desempeño en RPEC en estudiantes de diverso rendimiento, los participantes se seleccionaron de entre tres instituciones universitarias de diferente nivel de rendimiento en la prueba «Inicia». Las instituciones se clasificaron de acuerdo con sus puntajes promedio de logro en la prueba Inicia rendida en los años 2010 y 2011, en los niveles alto, medio y bajo. Para esto, se realizó un ranking de las universidades en base al puntaje Inicia de la prueba de conocimientos disciplinares. Para el nivel alto se seleccionó a una universidad que hubiera estado dentro de los primeros cinco lugares del ranking en ambos años y para el nivel bajo, a una universidad que hubiera estado dentro de los cinco últimos lugares del ranking en los dos años mencionados. Finalmente, para el nivel medio se 
seleccionó a una universidad que no cumpliera con los dos requisitos anteriores, es decir, que se encontrara dentro de los niveles medios de rendimiento en la prueba Inicia, agrupando siempre ambos años.

La distribución de la muestra se puede apreciar en la siguiente tabla:

Tabla 1: Distribución de la muestra

\begin{tabular}{|l|l|}
\hline Universidad & $\mathrm{N}^{\circ}$ \\
\hline Universidad Inicia alto & 24 \\
\hline Universidad Inicia medio & 35 \\
\hline Universidad Inicia bajo & 23 \\
\hline
\end{tabular}

\subsection{Procedimientos de toma de datos}

Los 82 participantes del estudio respondieron al instrumento denominado Reflexión Pedagógica en Base a Casos (RPEC) construido y validado durante un estudio piloto previo al trabajo que se reporta. Durante ese estudio preliminar se consultó la teoría, se realizó un proceso de juicio de experto con formadores de profesores nacionales e internacionales y un focus group con formadores de profesores nacionales, especialistas en didáctica de la lengua. Así también se aplicó el instrumento en tres rondas: dos tomas piloto con estudiantes comparables de otras dos universidades y una toma con profesores de lenguaje del sistema escolar que hubieran recibido la Asignación de Excelencia Pedagógica. De este proceso se derivan un reactivo, una rúbrica de RPEC y cuatro rúbricas de Lenguaje Académico (LA), de las cuales se reportan en este documento solo dos: metáforas gramaticales y organización del texto.

Con el objeto de verificar la relevancia en la formación inicial docente de la tarea que se solicitaría en el reactivo, durante el estudio piloto se aplicó una encuesta a 52 académicos pertenecientes a las universidades que participaron del estudio principal. Los resultados de esta encuesta revelan que analizar casos de enseñanza a partir de la teoría es una tarea frecuente entre los encuestados y que sus características son homólogas a las condiciones de evaluación que plantea el instrumento construido en este estudio. 
Las distintas fuentes que informan el instrumento RPEC y su rúbrica -teoría, estudiantes comparables, formadores de profesores nacionales e internacionales y profesores del sistema escolar-contribuyen a la construcción de un producto teóricamente actualizado, a la vez que adaptado a las exigencias y a la realidad de la formación de profesores en el país.

\subsubsection{El instrumento}

- Un video de 15 minutos que presenta los distintos momentos de una clase de Lenguaje y Comunicación en un cuarto básico, editados y etiquetados según su función, además de una diapositiva inicial que presenta el objetivo de la clase y un esquema de su estructura.

- Un reactivo que invita a escribir un análisis de caso a partir del video, utilizando para ello los distintos saberes adquiridos a lo largo de la formación inicial: disciplinarios, de didáctica general, de didáctica específica, de los contextos de la enseñanza, entre otros.

\subsubsection{La rúbrica RPEC}

En la Tabla 2 se resumen los criterios de evaluación de la rúbrica, asociados a las fuentes que las sustentan. Como se observa, se ha destacado el aporte de la teoría y de los expertos consultados durante el estudio piloto.

Tabla 2: Criterios de evaluación RPEC y fuentes

\begin{tabular}{|l|l|}
\hline $\begin{array}{l}\text { CRITERIOS DE EVALUACIÓN DE REFLEXIÓN PEDAGÓGICA EN } \\
\text { BASE A CASO }\end{array}$ & \multicolumn{1}{|c|}{ FUENTES } \\
\hline $\begin{array}{l}\text { A. Inferir razones o explicaciones que subyacen a las decisiones } \\
\text { pedagógicas de un profesor o profesora observado. }\end{array}$ & $\begin{array}{l}\text { Panel de expertos } \\
\text { Davis, 2006 } \\
\text { Schemp y Woorons, 2006 } \\
\text { Carter y Richardson, 1989 }\end{array}$ \\
\hline $\begin{array}{l}\text { B. Articular distintos dominios de conocimiento relevantes a la } \\
\text { enseñanza y el aprendizaje, en una reflexión escrita. }\end{array}$ & $\begin{array}{l}\text { Panel de expertos } \\
\text { Davis, 2006 } \\
\text { Carter y Richardson, 1989 } \\
\text { Silcock, 1994 } \\
\text { Luk (2008) } \\
\text { Lee (2005) }\end{array}$ \\
\hline
\end{tabular}




\begin{tabular}{|l|l|}
\hline $\begin{array}{l}\text { C. Sólido dominio de conceptos teóricos utilizados para analizar y } \\
\text { comprender la toma de decisiones pedagógicas. }\end{array}$ & $\begin{array}{l}\text { Panel de expertos } \\
\text { Darling Hammond y Bransford, } \\
2005\end{array}$ \\
& $\begin{array}{l}\text { Berliner, 1986 } \\
\text { Ben-Peretz, 2011 } \\
\text { Youngs y Bird, 2010 }\end{array}$ \\
\hline $\begin{array}{l}\text { D. Un tipo de análisis en el que se entregan fundamentos para las } \\
\text { afirmaciones, se consideran distintas perspectivas (por ejemplo, } \\
\text { distintas explicaciones para un fenómeno observado, o distintos } \\
\text { dominios de conocimiento relevantes), se evalúa y se ofrecen vías } \\
\text { alternativas de solución. En el nivel bajo, se trata de descripciones de } \\
\text { eventos y conductas; no se analiza la toma de decisiones, se entregan } \\
\text { opiniones sin fundamentos. }\end{array}$ & $\begin{array}{l}\text { Panel de expertos } \\
\text { Davis, 2006 }\end{array}$ \\
\hline
\end{tabular}

Sobre la base de los criterios de evaluación precedentes, la rúbrica gradúa en cinco niveles el desempeño de los estudiantes. En el nivel más alto se clasifican los textos cuyo propósito es analizar el sustento teórico de las decisiones pedagógicas de la profesora observada. Este análisis debe incluir al menos la reflexión respecto de tres de las siguientes dimensiones relevantes para la toma de decisiones pedagógicas: aprendices y aprendizaje, conocimiento sobre la asignatura, evaluación/calificación, enseñanza general y enseñanza específica. Cada una de estas dimensiones se explica en el texto usando conceptos teóricos precisos y correctos (por ejemplo, las preguntas abiertas promueven la generación de lectores activos), incluyendo modelos, enfoques y/o métodos de enseñanza (por ejemplo, cada una de estas estrategias corresponde a los tres momentos de la lectura) dentro de los cuales se inscriben las decisiones pedagógicas analizadas.

Los niveles intermedios se gradúan sobre la base de la cantidad de dimensiones relevantes para la toma de decisiones pedagógicas consideradas en la reflexión, al dominio teórico demostrado al analizar cada una de ellas y a la presentación de fundamentos para las afirmaciones realizadas. En el nivel más bajo se clasifica los textos cuyo propósito es solo describir la clase observada. Es posible que se haga mención a la enseñanza o al aprendizaje, pero como acciones realizadas por una profesora o por los niños, sin un claro intento por explicar el sustento teórico de la toma de decisiones pedagógica. 


\subsubsection{Las rúbricas de LA}

a) Metáforas gramaticales: esta rúbrica explora la capacidad de los estudiantes para utilizar conceptos abstractos en posición de sujeto gramatical, lo que permite predicar o decir algo sobre dichos conceptos (Schleppegrel y Oliveira, 2006; Snow y Ucelli, 2008). El uso de este recurso no solo demuestra un dominio discursivo, sino también el dominio disciplinario. La escala se gradúa en cuatro niveles de acuerdo con la presencia de tres, dos, uno o ninguno de los tres tipos de metáforas: nominalizaciones en las que conceptos abstractos están utilizados como agentes (ejemplo: «el enfoque comunicativo es abordado por la profesora...»), verbos de proceso que aparecen sustantivados y funcionan como sujeto (ejemplo: «la lectura de la profesora...», en lugar de «la profesora lee...») y categorías abstractas (hiperónimos) usados para agrupar secuencias referidas anteriormente (ejemplo: «el fenómeno recién descrito...», «este proceso observado...»).

b) Organización del texto: esta rúbrica explora la capacidad de los estudiantes para imponer una organización explícita al texto (Ucelli, Dobbs y Scott en prensa) sobre la base de los conceptos teóricos incluidos en el análisis. Se asume que el desempeño en esta escala no solo involucra dominio discursivo sino que también dominio disciplinario (Maclellan, 2008). En efecto, la capacidad para imponer una estructura a un texto académico depende no solo del dominio de estructuras convencionales y de marcadores discursivos, sino que también de los conceptos incluidos y de sus interrelaciones. En el nivel más alto (nivel 4) se clasifican los textos cuyo contenido se organiza en secciones que corresponden a conceptos teóricos (ejemplo: cada sección corresponde a uno de los momentos de la lectura). En el nivel 3 se clasifican los textos organizados en base a conceptos técnicos (ejemplo: una sección referida al clima del aula, otra respecto de la lectura en voz alta, etc.), pero de manera fragmentaria, de modo que no se organiza la totalidad del texto, sino que se suman secciones de un modo no planificado. En los niveles 2 y 1 se clasifican los textos que no ofrecen evidencia de una capacidad para imponer una organización planificada al texto, pues se limitan a imitar, de manera más o menos rigurosa, la estructura del reactivo (nivel 2) o la cronología del video observado (nivel 1). 


\subsubsection{Condiciones de evaluación}

La toma de datos se llevó a cabo en una modalidad de aplicación grupal. Se solicitó la cooperación a los profesores de cuarto año de carrera de las instituciones seleccionadas, de modo que cedieran un módulo de clases durante el cual los estudiantes respondieran el instrumento. Todos los estudiantes participantes fueron informados de los objetivos del estudio y todos firmaron un consentimiento que autorizaba el uso de sus respuestas con fines de investigación.

Las aplicaciones grupales comenzaron con la observación (dos veces o más, según necesidad declarada por los estudiantes) del video a analizar. Durante la observación se les solicitó tomar notas, para luego pasar al análisis individual solicitado en las instrucciones. En todas las aplicaciones, una de las investigadoras estuvo presente en la sala de clases durante todo el proceso para explicar el objetivo de la investigación, dar las instrucciones, mostrar el video y resolver dudas.

\section{Procedimientos de análisis de datos}

Los datos recogidos en el estudio que se reporta fueron codificados con el objeto de medir dos constructos: reflexión pedagógica en base a casos y lenguaje académico. El objetivo fue contar con medidas susceptibles de ser comparadas en la búsqueda de relaciones entre el dominio de lenguaje académico y la calidad de la reflexión pedagógica. Los esquemas de codificación -de reflexión pedagógica en base a casos y las dos rúbricas de lenguaje académico que se reportan- fueron ambos piloteados antes de su aplicación a la muestra definitiva.

En este estudio se utilizaron rúbricas que permitieron valorar cada una de las dimensiones a evaluar, de modo de contar con una medida objetiva que reflejara niveles de logro para cada dimensión. Cada dimensión de la rúbrica RPEC fue puntuada con niveles de 1 a 5 y cada nivel de la rúbrica LA fue puntuada con un rango de 1 a 4 , lo que posibilita realizar análisis estadísticos de comparación de promedios.

Se realizaron distintos análisis estadísticos con los datos obtenidos: 


\section{a) Análisis diferenciado de los datos según universidad de} origen: análisis descriptivo y comparación de promedios. Para este último se aplicó una prueba de análisis de varianza (ANOVA) de un factor (universidad de origen) a los promedios de RPEC y a las medidas de LA.

b) Regresión estadística: que permitió evaluar la hipótesis de que el dominio de lenguaje académico explica la capacidad de razonamiento pedagógico.

La codificación se realizó con dos asistentes de investigación con conocimientos de didáctica de la lengua y formación de profesores. Para asegurar la confiabilidad, durante la codificación se consideraron dos instancias de doble codificación: al inicio y en mitad del proceso. Respecto de las rúbricas de LA, en la primera ronda se midió la consistencia con un $\mathrm{n}=14$ y se obtuvieron coeficientes Kappa de 0,87 para la medida «metáforas» y 1 para la medida «organización» $(\mathrm{p}<0.05)$. La segunda ronda de doble codificación se realizó con un $\mathrm{n}=10$ y se obtuvieron coeficientes Kappa de 0,61 para «metáforas» y 0,64 significativo para organización $(\mathrm{p}<0.05)$. Respecto de la rúbrica $\mathrm{RPEC}$, en la primera ronda con un $\mathrm{n}=15$ se obtuvo un coeficiente Kappa de 0,61 ( $<<0.01)$ y en la segunda ronda, con un $n=13$, se obtuvo un coeficiente Kappa significativo de 0,51 $(\mathrm{p}<0.05)$.

\section{Resultados}

Los resultados del estudio se presentan divididos en tres secciones: lenguaje académico, reflexión pedagógica en base a casos y relaciones entre ambas medidas. Todos estos análisis se realizaron con la muestra total de participantes, $n=82$. En cada caso se entregan primero los resultados promedio y luego los desagregados por institución participante.

\subsection{Resultados lenguaje académico}

En la tabla siguiente se puede observar la media obtenida para cada dimensión (de una escala de valores entre el 1 y el 4) y también la desviación estándar, considerando la totalidad de la muestra. En 
los gráficos se puede observar en detalle la frecuencia con que se codificaron las muestras en cada nivel de las rúbricas.

Tabla 3: Descriptivos para cada dimensión de análisis de LA

\begin{tabular}{|l|c|c|}
\hline Dimensión de análisis & Media & DS \\
\hline Metáforas gramaticales & 1,97 & 0,75 \\
\hline Organización & 2,32 & 0,45 \\
\hline
\end{tabular}

Gráficol: Frecuencia de los niveles de calificación rúbrica «metáforas gramaticales»

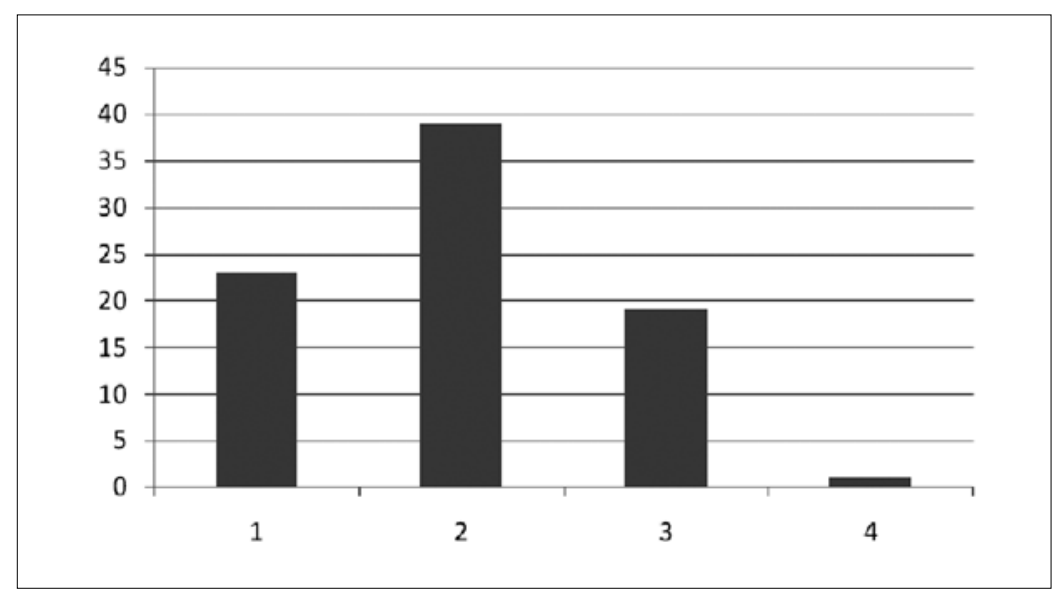

Este gráfico muestra que el valor 2 es la calificación más frecuente para esta dimensión. Por su parte, mientras los valores 1 y 3 están prudentemente presentes, el valor 4 (de mayor dificultad) tiene una baja frecuencia de aparición. Lo anterior estaría señalando que los estudiantes no demuestran capacidad para utilizar los tres tipos de metáforas gramaticales en estudio. La mayoría solo puede utilizar un tipo de metáfora (nivel 2) y cerca del 20\% de ellos solo puede utilizar dos tipos. Deriva de la aplicación de la rúbrica la evidencia anecdótica de que el tipo de metáfora más frecuente sería el uso de conceptos abstractos en posición de sujeto oracional (ejemplo: la clase, la lectura, las reglas de convivencia) y las menos frecuente serían las categorías teóricas que refieren a porciones anteriores en el texto (ejemplo: el fenómeno descrito, el proceso señalado). 
Gráfico 2: Frecuencia de niveles de calificación en la rúbrica «organización»

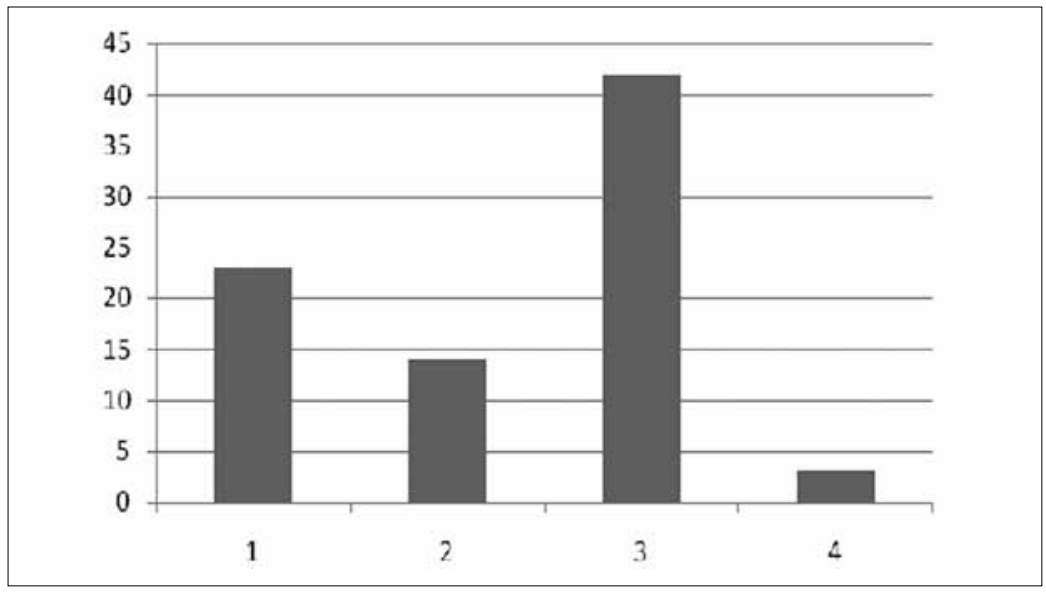

En el caso de la dimensión «organización», se puede observar que si bien todos los niveles de calificación están representados, esta es una dimensión de alto nivel de dificultad, pues el nivel superior es el que se utiliza con menor frecuencia. Los resultados señalan que la mayoría de las muestras se agrupa en torno al nivel 3, que corresponde a una organización basada en distintos focos temáticos (ejemplo: el clima de aula, el manejo disciplinario, etc.) que no impone una estructura a la totalidad del texto, sino que se presenta como secciones fragmentarias que no parecen provenir de una planificación. Una importante proporción de los estudiantes se agrupa en torno a los niveles 1 y 2 que tienen en común que no revelan una capacidad para imponer una estructura al texto, sino que se valen de la estructura de la realidad (en este caso, de la narrativa del video) o del reactivo.

Al analizar los resultados separando a los estudiantes según universidad de origen, se observa que en la medida «metáforas gramaticales» los resultados se organizan de mayor a menor desde el grupo Inicia alto al bajo, como se observa en la tabla que sigue. El caso es diferente para la medida «organización» en el que la secuencia de puntajes promedio no reproduce un orden según puntajes Inicia de la institución a la que pertenecen los estudiantes. 
Tabla 4: Medias de medición LA de acuerdo con universidad de origen

\begin{tabular}{|l|c|c|c|}
\hline Medidas de LA & $\begin{array}{c}\text { Universidad Inicia } \\
\text { alto }\end{array}$ & $\begin{array}{c}\text { Universidad Inicia } \\
\text { medio }\end{array}$ & $\begin{array}{c}\text { Universidad Inicia } \\
\text { bajo }\end{array}$ \\
\hline Metáforas gramaticales & 2,25 & 1,88 & 1,82 \\
\hline Organización & 2,29 & 2,51 & 2 \\
\hline
\end{tabular}

Según los resultados obtenidos de la comparación de promedios entre grupos de estudiantes, se verifican diferencias unilaterales en el caso de la medida de «metáforas» $(\mathrm{F}=2,37, \mathrm{gl}=2, \mathrm{p}<0,05)$ y en el margen de la significancia para la medida de «organización» $(\mathrm{F}=2,21$; $\mathrm{gl}=2, \mathrm{p}=0,05)$. Específicamente, respecto de la medida «metáforas gramaticales», se presentaron diferencias significativas unilaterales entre la universidad de Inicia alto (mayor promedio) y la de Inicia medio $(\mathrm{t}=1,96, \mathrm{gl}=57, \mathrm{p}<0,02)$ y entre la de Inicia alto (mayor promedio) e Inicia bajo $(\mathrm{t}=-1,98, \mathrm{gl}=45, \mathrm{p}<0,03)$. Por último, en la medida de «organización» la única diferencia significativa se ubicó al comparar el mayor promedio de la universidad de Inicia medio con la universidad de Inicia bajo $(\mathrm{t}=-2,19, \mathrm{gl}=56, \mathrm{p}<0,03)$.

\subsection{Resultados RPEC}

En una escala de 1 a 5, los estudiantes de la muestra total obtienen una media de 2,6 y una desviación típica de 0,81. Este dato señala que, en promedio, la totalidad de los estudiantes tiende hacia RPEC en las que no se utilizan conocimientos teóricos precisos y correctos para analizar las decisiones pedagógicas de la profesora observada. En este nivel se ubican textos en que abundan los errores conceptuales y las explicaciones no técnicas, o que recurren a conocimiento de dominio general.

Respecto de la distribución de los resultados obtenidos, se observa que la rúbrica RPEC tiene un comportamiento similar a lo normal, como se observa en el siguiente gráfico. 
Gráfico 3: Frecuencia de niveles de calificación en la rúbrica RPEC

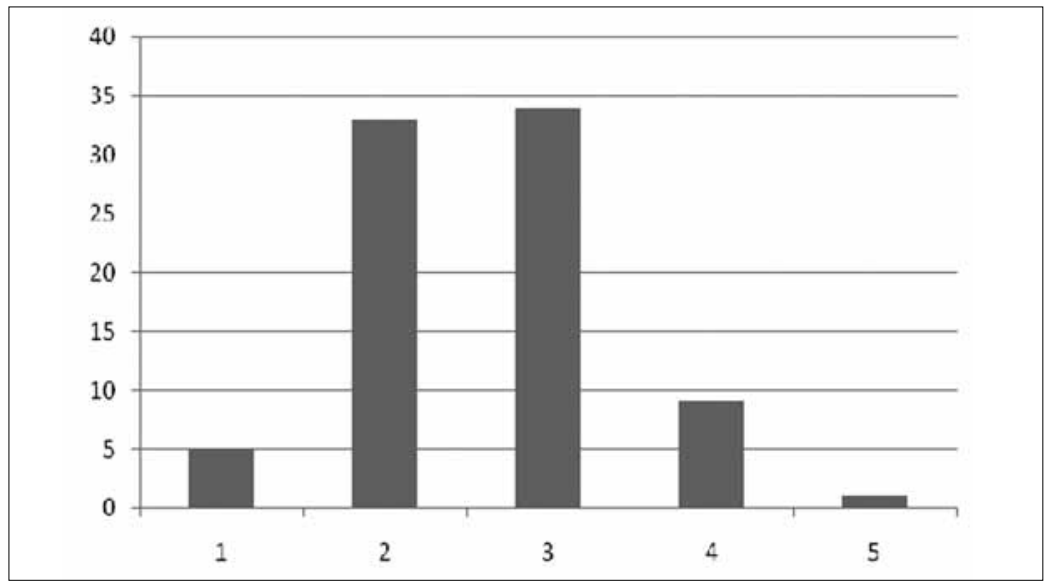

\section{A continuación se ofrecen ejemplos que ilustran los niveles de desempeño en RPEC:}

\begin{tabular}{|c|c|}
\hline $\begin{array}{l}\text { Ejemplos para ilustrar los procedimientos de } \\
\text { codificación en RPEC }\end{array}$ & Justificación de la codificación \\
\hline RPEC: nivel l (fragmento de muestra) & \multirow{2}{*}{$\begin{array}{l}\text { Se entrega una descripción y no un análisis. } \\
\text { Se refiere a la enseñanza, pero como acciones } \\
\text { realizadas por una profesora, sin un claro intento } \\
\text { por explicar la toma de decisiones. }\end{array}$} \\
\hline $\begin{array}{l}\text { La docente enseña primero mostrando unas imágenes } \\
\text { a los alumnos y les pide que hagan predicciones } \\
\text { acerca de ellas. Luego les lee un cuento y les da una } \\
\text { pequeña introducción diciendo de dónde es el libro, } \\
\text { la editorial, etc. Luego de leer el cuento les hace } \\
\text { preguntas a los alumnos. (G3, M3) }\end{array}$ & \\
\hline RPEC: nivel 2 (fragmento de muestra) & \multirow[b]{2}{*}{$\begin{array}{l}\text { El análisis articula dos dimensiones relevantes a } \\
\text { la enseñanza y el aprendizaje, a saber: aprendices } \\
\text { y aprendizaje e instrucción. Las explicaciones no } \\
\text { revelan dominio de la teoría que subyace a la } \\
\text { clase. Se introducen errores conceptuales («una } \\
\text { clase magistral en la cual ella es la mediadora y } \\
\text { el hilo conductor...») y se ofrecen explicaciones } \\
\text { generales, no técnicas («...otra dinámica en la } \\
\text { que todos se sintieran parte de él...»; «...tips } \\
\text { y énfasis a cómo identificar realmente la idea } \\
\text { principal del cuento.» }\end{array}$} \\
\hline $\begin{array}{l}\text { La profesora realiza una clase magistral en la cual } \\
\text { ella es la mediadora y el hilo conductor de la clase. } \\
\text { Debido al tema de la clase me parece que cumple } \\
\text { positivamente con tener la atención de los niños, } \\
\text { aunque quizás se podría haber leído el cuento con } \\
\text { otra dinámica en la que todos se sintieran parte de } \\
\text { él (en un círculo por ejemplo). } \\
\text { La profesora podría dar más tips y énfasis a cómo } \\
\text { identificar realmente la idea principal del cuento. } \\
(\mathrm{G} 3, \mathrm{M} 4)\end{array}$ & \\
\hline
\end{tabular}




\begin{tabular}{|c|c|}
\hline PEC: nivel 3 (fragmento de muestra) & \multirow[b]{2}{*}{$\begin{array}{l}\text { Se presenta un análisis que intenta explicar las } \\
\text { decisiones pedagógicas observadas y que articula } \\
\text { tres de las dimensiones relevantes a la enseñanza } \\
\text { y al aprendizaje: instrucción, aprendices y } \\
\text { aprendizaje y conocimiento sobre la asignatura. } \\
\text { Si bien el análisis involucra conocimiento } \\
\text { teórico («conocimientos previos», «estructura } \\
\text { del cuento», "predicciones») las explicaciones } \\
\text { tienden a ser generales en lugar de técnicas ( «... } \\
\text { relaciones entre sus conocimientos previos con } \\
\text { el tema...», «con el propósito de introducir el } \\
\text { contenido...»; «...que los estudiantes realicen } \\
\text { predicciones en las que participen todos los } \\
\text { alumnos...»; «....identificar la idea principal del } \\
\text { texto que es una muy buena estrategia»). } \\
\end{array}$} \\
\hline $\begin{array}{l}\text { El docente pretende establecer relaciones entre } \\
\text { sus conocimientos previos con el tema que se } \\
\text { abarcará (cuento), con el propósito de introducir } \\
\text { el contenido. Para ello se realizan preguntas en las } \\
\text { que los estudiantes reconocen durante la clase la } \\
\text { estructura del texto (cuento), con el propósito de que } \\
\text { realicen predicciones en las que participen todos y } \\
\text { así identificar la idea principal del texto que, es una } \\
\text { muy buena estrategia. (G2, M1) }\end{array}$ & \\
\hline RPEC: nivel 4 (fragmento de muestra) & \multirow[b]{2}{*}{$\begin{array}{l}\text { Se presenta un análisis que intenta explicar las } \\
\text { decisiones pedagógicas observadas y que articula } \\
\text { tres de las dimensiones relevantes a la enseñanza } \\
\text { y al aprendizaje: instrucción, aprendices y } \\
\text { aprendizaje y conocimiento sobre la asignatura. } \\
\text { Las explicaciones incluyen conceptos teóricos } \\
\text { que se utilizan de manera precisa y técnica } \\
\text { (aprendizajes significativos, discurso extendido). } \\
\text { Se presentan evidencias como fundamento para } \\
\text { las afirmaciones. }\end{array}$} \\
\hline $\begin{array}{l}\text { Las estrategias que utiliza están vinculadas a los } \\
\text { principios generales de la didáctica, uno de los } \\
\text { cuales corresponde a los aprendizajes significativos. } \\
\text { Este principio se utiliza para que los alumnos se } \\
\text { involucren con el aprendizaje desde un punto de } \\
\text { vista propio y un significado personal. La profesora lo } \\
\text { pone en juego cuando les pide a los alumnos realizar } \\
\text { conexiones entre el cuento y acontecimientos de su } \\
\text { propia vida. Esto les ayuda a comprender mejor el } \\
\text { cuento y así poder identificar el tema o idea principal } \\
\text { (...); otra estrategia que utiliza es la que consiste en } \\
\text { desarrollar el discurso extendido en la oralidad de } \\
\text { los alumnos (...), (G2, M22) }\end{array}$ & \\
\hline RPEC: nivel 5 (frag & \\
\hline $\begin{array}{l}\text { De esta forma podemos concluir que la docente } \\
\text { posee estrategias claras para enseñar comprensión } \\
\text { de lectura (...) junto con que es capaz de dividir } \\
\text { la lectura en tres grandes momentos, comenzando } \\
\text { con predicciones antes de esta, posteriormente } \\
\text { realizando preguntas y relacionando los párrafos y, } \\
\text { por último, realizar una comprensión global de lo } \\
\text { narrado y relacionando con los otros dos momentos } \\
\text { (...); la forma en la que la docente presenta la clase } \\
\text { correspondería al enfoque comunicativo, ya que ella } \\
\text { hace que los estudiantes participen en situaciones } \\
\text { reales de lectura, siendo capaces de darle un sentido } \\
\text { y relacionarlo con sus entornos más próximos (...); } \\
\text { además, evidencia saber que debe enseñar desde un } \\
\text { género discursivo. (G2, MP) }\end{array}$ & $\begin{array}{l}\text { Se presenta un análisis que intenta explicar las } \\
\text { decisiones pedagógicas observadas y que articula } \\
\text { tres de las dimensiones relevantes a la enseñanza } \\
\text { y al aprendizaje: instrucción, aprendices y } \\
\text { aprendizaje y conocimiento sobre la asignatura } \\
\text { El análisis involucra conocimiento teórico, no solo } \\
\text { explicado de forma precisa y técnica, sino que } \\
\text { incluye además modelos, enfoques o métodos de } \\
\text { enseñanza (los tres momentos de la lectura y e } \\
\text { enfoque comunicativo). Se presentan evidencias } \\
\text { como fundamento para las afirmaciones. }\end{array}$ \\
\hline
\end{tabular}

\section{La siguiente tabla y gráfico representan los resultados obtenidos de la aplicación de la rúbrica RPEC a los estudiantes de las tres instituciones participantes.}


104 REFLEXIÓN PEDAGÓGICA EN BASE A CASOS Y DOMINIO DE LENGUAJE ACADÉMICO EN ESTUDIANTES DE CUARTO AÑO DE PEDAGOGÍA EN EDUCACIÓN BÁSICA - S. Concha, C. Hernández, F. del Río, F. Romo, L. Andrade

Tabla 5: Medias de medición de RPEC de acuerdo con la universidad de origen

\begin{tabular}{|l|c|c|c|}
\hline & Universidad Inicia alto & Universidad Inicia medio & Universidad Inicia bajo \\
\hline Reflexión pedagógica & 2,54 & 3,14 & 1,86 \\
\hline
\end{tabular}

Gráfico 4: Distribución de puntajes RPEC por universidad de origen

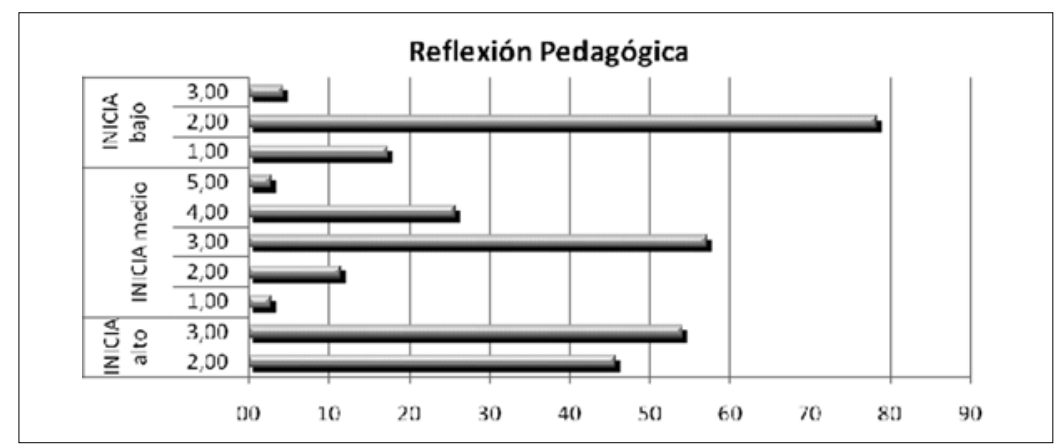

Los cálculos de comparación entre grupos para esta medida muestran diferencias significativas (bilaterales) $(\mathrm{F}=29,01, \mathrm{gl}=2$, $\mathrm{p}<0,00)$. La universidad de Inicia medio presentó un logro promedio significativamente mayor que la universidad de Inicia alto $(t=-3,34$, $\mathrm{gl}=57, \mathrm{p}<0,01$ ), y a su vez, la universidad de Inicia alto presentó un logro promedio significativamente mayor que la universidad de Inicia bajo $(t=4,75, g l=45, p<0,00)$.

Como se observa en el gráfico de frecuencias, en promedio el grupo Inicia alto realizó un análisis que se caracterizó por su falta de dominio teórico. Más del $50 \%$ de ellos produjo un análisis que si bien aborda al menos dos de las dimensiones relevantes para la toma de decisiones pedagógica, no las analiza de manera técnica, sino general (nivel 3). Otro grupo importante de esos participantes (más del 40\%) se ubicó en el nivel 2 que corresponde a textos en los que se incluyen errores conceptuales, a la vez que explicaciones generales o no técnicas respecto de las dimensiones relevantes para la toma de decisiones pedagógicas.

Los estudiantes del grupo Inicia bajo se agrupan en gran proporción (casi un 80\%) en el nivel 2 ya descrito, además de una proporción menor (cercana al 20\%) de estudiantes clasificados en el nivel 1 , caracterizado por textos centrados en la mera descripción de 
las acciones realizadas por la profesora y/o por los niños en la clase observada.

El promedio más alto de desempeño en esta rúbrica lo obtienen los estudiantes de la institución Inicia medio. Se observa que en este grupo los puntajes se distribuyen mejor en los distintos niveles de la rúbrica, de modo que no obstante la mayor parte de ellos (más de 50\%) se clasifica en el nivel 3, más del 20\% alcanza el nivel 4 caracterizado por análisis que explican las decisiones pedagógicas de la profesora desde al menos dos de las dimensiones relevantes para la pedagogía, utilizando para ello conceptos teóricos precisos y correctos y fundamentos para las afirmaciones. Hay incluso en este grupo un porcentaje muy menor de estudiantes en el nivel 5 que se diferencia del 4 en que el análisis puede abordar al menos tres de las dimensiones y la teoría que se utiliza asciende a un nivel más amplio de enfoques o métodos de enseñanza.

\subsection{Relaciones entre las variables RPEC y LA}

Para contrastar la hipótesis de la existencia de una relación entre el dominio de lenguaje académico y la capacidad de construir una reflexión pedagógica en base a casos, se llevaron a cabo dos tipos de análisis. En primer lugar se calcularon los coeficientes de correlación bivariada entre la medida de «reflexión pedagógica» y las medidas de «lenguaje académico». Los resultados mostraron que la medida de «reflexión pedagógica» solo correlacionaba de manera significativa con la medida de «organización» $(\mathrm{r}=0,406, \mathrm{p}=0,001)$.

En segundo lugar se llevó a cabo la prueba de regresión lineal, con el fin de encontrar el mejor modelo que explicara la medida de «reflexión pedagógica». En esta prueba se utilizaron varios modelos de ecuaciones, partiendo con una donde se incluyeron ambas medidas de LA, para luego buscar entre las combinaciones posibles la solución de mayor parsimonia. Los resultados señalan que el mejor modelo es aquel en el que la medida de «organización» explica el 17\% $\left(R^{2}=0,171\right)$ de la varianza de la medida de «reflexión pedagógica», con una relación lineal significativa $(F=15,81, p=0,00)$. 
106 REFLEXIÓN PEDAGÓGICA EN BASE A CASOS Y DOMINIO DE LENGUAJE ACADÉMICO EN ESTUDIANTES DE CUARTO AÑO DE PEDAGOGÍA EN EDUCACIÓN BÁSICA - S. Concha, C. Hernández, F. del Río, F. Romo, L. Andrade

\section{Conclusiones}

Las conclusiones del estudio se organizan en relación con las preguntas de la investigación.

¿Qué capacidad tienen los estudiantes de cuarto año de Pedagogía en Educación Básica para reflexionar pedagógicamente sobre la base de casos, en modalidad escrita?

En la medida RPEC, la media de todos los participantes es de 2,6 en una rúbrica de cinco niveles. Lo anterior significa que, respecto del caso observado en el estudio y sobre la base de los criterios de evaluación aplicados, los estudiantes tendieron a producir textos en los que reflexionaban respecto de la toma de decisiones pedagógicas de la profesora observada, pero no desde un dominio teórico sólido, sino desde un punto de vista general, no técnico, como de sentido común. En el nivel 2 en el que se clasifica la mayoría de los estudiantes, abundan los errores conceptuales y las expresiones en las que se mencionan palabras técnicas en contextos en los que no es posible determinar si el estudiante comprende o no su significado.

¿Qué dominio de lenguaje académico demuestran los estudiantes de cuarto año de Pedagogía en Educación Básica, al producir reflexiones pedagógicas sobre la base de casos en modalidad escrita?

En el presente artículo se reportan los resultados obtenidos para las medidas «metáforas gramaticales» y «organización». Respecto de la primera medida, los resultados promedio se ubican en torno al nivel 2 en una escala de 4 niveles, lo que refleja que la mayoría de los participantes solo utiliza un tipo de estas metáforas que, según la literatura revisada, deberían caracterizar a la escritura de un experto disciplinario que ubica en posición de sujeto gramatical distintos conceptos abstractos para referirse a ellos. Respecto de la segunda medida, el promedio del total de la muestra se ubica también en el nivel 2 de una escala de 4 niveles. Lo anterior señalaría que, en promedio, los participantes tendieron a producir textos en los que no impusieron una organización de la información guiada por las categorías teóricas involucradas en su análisis. Más bien imitaron la 
estructura del reactivo, en una estrategia que no refleja planificación ni gestión intencionada de la información con un propósito relacionado con el análisis.

¿Hay alguna relación entre la capacidad para reflexionar pedagógicamente sobre la base de casos, en modalidad escrita, y el dominio de lenguaje académico de estudiantes de cuarto año de Pedagogía en Educación Básica?

Los resultados del estudio solo permiten confirmar la relación entre la capacidad para producir por escrito una RPEC y la capacidad para imponer una organización explícita a la información incluida en el texto. Específicamente, en este estudio se observa una correlación entre la capacidad de reflexionar pedagógicamente por escrito y la capacidad para organizar un texto sobre la base de conceptos teóricos que forman parte de dicha reflexión. Lo anterior se entiende como un dominio discursivo y disciplinario, que es característico de la escritura de registro académico.

¿Hay diferencias en la capacidad de reflexionar pedagógicamente en base a casos y en el dominio de lenguaje académico entre estudiantes de cuarto año de Pedagogía en Educación Básica de instituciones con promedios en prueba Inicia alto, medio y bajo?

Los resultados obtenidos para la medida RPEC no reproducen la secuencia de los promedios Inicia (2010 y 2011) de las instituciones de los estudiantes en estudio. En efecto, los mayores puntajes en RPEC los obtiene la universidad de desempeño Inicia medio; en segundo lugar se ubican los estudiantes de la institución con Inicia alto y, en tercer lugar, los estudiantes de la institución con Inicia bajo. Hay diferencias significativas tanto entre el resultado obtenido por el grupo de estudiantes de la universidad Inicia medio y el de la universidad Inicia alto, como entre los participantes de la universidad alta y la baja.

En el caso de las medidas de LA, los resultados son más coherentes con la secuencia de puntajes Inicia, no obstante no se reproducen con exactitud. En efecto, para la medida «metáforas gramaticales» el grupo de participantes de la Universidad Inicia alto 
obtiene un puntaje significativamente mayor que el de las instituciones Inicia medio y bajo. En el caso de la medida «organización del texto», la única diferencia significativa se da entre el promedio obtenido por la universidad Inicia medio y la de Inicia bajo.

\section{Discusión}

Hay coincidencia en la literatura respecto de que los profesores novatos deberían ser capaces de reflexionar sobre eventos del aula, como parte de su proceso de convertirse en profesores expertos (Berliner, 1986; Darling Hammond y Bransford, 2005; Carter y Richardson, 1989; Schemp y Woorons, 2006; Davis, 2006). Según la literatura revisada para este estudio, se trataría de la capacidad de analizar situaciones de aula sobre la base de distintos saberes. Considerando este principio sustentado en la literatura disponible, los bajos resultados obtenidos en este estudio preocupan por dos razones. En primer lugar, porque se ha evaluado a estudiantes de cuarto año de Pedagogía en Educación Básica, que difícilmente podrán tener más oportunidades formativas para aprender a realizar este ejercicio, en circunstancias que se reconoce la relevancia de ello para alcanzar la experticia en la enseñanza. En segundo lugar, porque señalan un posible problema en los programas formativos.

Respecto de lo último, es difícil sostener a partir de una investigación como esta que los estudiantes en estudio no posean los conocimientos relevantes para analizar las distintas dimensiones de un aula. Sería necesaria una prueba de contenidos para saberlo. Lo que sí revelan estos resultados es que los estudiantes no demostraron capacidad para utilizar esos conocimientos en un análisis como el solicitado. Lo anterior demanda una revisión de la forma en que se presentan estos saberes a los futuros profesores durante su formación, pues según los datos aquí recogidos, las metodologías de enseñanza no permitirían que los estudiantes se representen los contenidos de modo que estén disponibles para este tipo de análisis. Es interesante que los resultados de la encuesta realizada a los formadores de profesores de las instituciones participantes señalen que la tarea solicitada es parte de sus programas formativos. Así, los resultados de este estudio estarían indicando una discontinuidad entre lo que 
se declara que se enseña y los desempeños demostrados por los estudiantes.

Si bien hay escasa evidencia disponible, se propone que la aplicación sistemática de ejercicios de reflexión pedagógica escrita durante la formación inicial podría lograr, gradualmente, mejorar las capacidades de los estudiantes en esta tarea (Fund et al., 2002). En este sentido, los formadores de profesores podrían proveer a sus estudiantes de un modelo y de criterios de evaluación que definan con claridad qué se entiende por RPEC y fijen respecto de ella niveles de logro, además de las retroalimentaciones que sería necesario hacer recursivamente para impulsar este desarrollo. Más aún, y como se ha dicho ya en este informe, las investigadoras derivan en parte este trabajo de aquellos estudios que proponen que una manera de velar para que los estudiantes puedan utilizar activa y articuladamente los distintos conocimientos adquiridos durante su formación para un análisis de caso de aula, es que los señalados conocimientos sean enseñados en torno a casos (Carter y Richardson, 1989; Kim y Hannafin, 2008) y no solo evaluados en relación a ellos.

Si bien la variable puntajes Inicia se introduce en este estudio para velar por una muestra diversa respecto de su rendimiento, los resultados obtenidos merecen una reflexión. Aun cuando es necesario tener más datos y una exploración sistemática de lo observado aquí, llama la atención que los resultados en RPEC no se organicen de manera equivalente a los resultados Inicia 2010 y 2011 de las instituciones seleccionadas. Es importante destacar que los participantes se seleccionaron en relación con puntajes promedio de una prueba de saberes disciplinarios enfocada en si los estudiantes saben o no esos contenidos, y no si saben utilizarlos, como el instrumento RPEC requiere. Al respecto, será interesante este estudio como antecedente de los resultados que se verifiquen en las futuras aplicaciones de Inicia en que se ha introducido ya no solo el reconocimiento, sino el análisis y aplicación de los saberes disciplinarios evaluados. Lo anterior tiene relevancia como reflejo de los contenidos incluidos en las mallas curriculares y de las metodologías de formación inicial docente de las instituciones evaluadas, como se ha discutido ya en este artículo. En efecto, instrumentos a gran escala como Inicia y otros de aplicación 
interna en los programas de formación inicial docente, deberían poder demostrar lo más fielmente qué y cómo se ha enseñado a los estudiantes para ser profesores, en lugar de ofrecer resultados que reflejen su nivel socioeconómico o imiten la distribución de sus puntajes PSU de entrada, como ha ocurrido con Inicia hasta el momento.

Los resultados de este estudio dialogan con lo propuesto en la literatura respecto de que es posible construir instrumentos válidos y confiables para ser utilizados al interior de la formación inicial docente (FID) para evaluar reflexión pedagógica (Fund et al., 2002; Youngs y Birds, 2010). Los métodos utilizados en esta investigación sugieren que es posible derivar criterios de análisis sistemáticos a partir de un modelo de reflexión pedagógica y que la escritura es un medio privilegiado para realizar este tipo de análisis, pues provee de un testimonio de esta capacidad (deja de ser una habilidad interna, solo mental). Adicionalmente, según se propone en la literatura revisada, analizar la reflexión pedagógica por escrito haría posible evaluar su desarrollo y enseñarla (Davis, 2006; Lee, 2005; Luk, 2008; Fund et al., 2002; Youngs y Birds, 2010). En efecto, este estudio contribuye a la noción de que una manera de aprender a ser profesor, en este caso de aprender a reflexionar pedagógicamente como un profesor experto, es por medio de la escritura, en una lógica de alfabetización pedagógica (Mclellan, 2008).

$\mathrm{Al}$ respecto, sin embargo, este estudio confirma lo observado por Luk (2008) respecto de que las habilidades de producción escrita de los estudiantes interactúan con su capacidad para producir una reflexión pedagógica por escrito. Los datos aquí recogidos señalan que la calidad de la reflexión pedagógica producida está relacionada con la habilidad para organizar discursivamente un texto. Lo anterior es muy interesante, puesto que confirma la estrecha relación que existe entre el conocimiento teórico y el dominio discursivo en el registro académico (Snow y Uccelli, 2009; Schleppegrel y Oliveira, 2006). En efecto, parece ser que ninguno de los dos ámbitos en solitario basta para realizar esta compleja tarea, sino que se necesita un dominio que articule ambas dimensiones. Tal como propusiera Berliner (1986), un profesor experto logra inferir de una sala de clases categorías teóricas que sustentan la toma de decisiones pedagógicas. El estudio que se 
describe complementa la observación de Berliner con evidencia que señala que cuando esa reflexión pedagógica se realiza por escrito, no requiere solamente de la capacidad de usar los conocimientos teóricos para analizar el aula, sino también de un dominio discursivo que permita organizar un texto en base a esas categorías.

Preocupan, en este sentido, los resultados derivados de la dimensión tipo de organización de la rúbrica LA, pues señalan que, en promedio, prácticamente la mitad de los estudiantes en estudio utiliza una estrategia ingenua para organizar sus textos que solo considera el nivel del contenido (qué decir) y no el nivel retórico (cómo decir), como proponen Bereiter y Scardamalia (1987). Esta estrategia improvisada o no planificada replica estudios previos realizados por las investigadoras (Concha, Aravena, Coloma y Romero et al, 2010; Concha y Paratore, 2011) con estudiantes del sistema escolar, que estarían reflejando una debilidad en la formación en producción escrita de los estudiantes universitarios en estudio, que pudiera estarse arrastrando desde la experiencia escolar.

Similar es la situación de las metáforas gramaticales, que serían testimonio de un dominio discursivo y teórico (Snow y Uccelli, 2009) que no se confirma en estos datos. Al respecto, es posible que la simpleza promedio de los análisis recogidos estuviera relacionada con estos resultados, en tanto no se haría necesario utilizar distinto tipo de metáforas gramaticales si no se actualizan suficientes conceptos teóricos.

\section{Referencias}

Barksdale-Ladd, M., Draper, M., King, J. Oropallo, K. y M. Radencich, (2001) «Four approaches to preservice teachers' involvement in the writing of case stories: a qualitative research project» en Teaching and Teacher Education. 17, pp. 417-431.

Ben-Peretz, M., (2011) «Teacher knowledge: What is it? How do we uncover it? What are its implications for schooling?» en Teaching and Teacher Education. 27 (1), pp. 3-9.

Bereiter, C. y M. Scardamalia, (1987) The psychology of written composition. Hillsdale, N.J.: Lawrence Erlbaum.

Berliner, D., (1986) «In pursuit of the expert pedagogue» en Educational Researcher. 15 (7), pp. 5-13. 
112 REFLEXIÓN PEDAGÓGICA EN BASE A CASOS Y DOMINIO DE LENGUAJE ACADÉMICO EN ESTUDIANTES DE CUARTO AÑO DE PEDAGOGÍA EN EDUCACIÓN BÁSICA - S. Concha, C. Hernández, F. del Río, F. Romo, L. Andrade

Borko, H. y C. Livingston, (1989) «Cognition and improvisation: differences in mathematics instruction by expert and novice teachers» en American Educational Research Journal, 26 (4), pp. 473-498.

Braun, J. y T. Crumpler, (2003) «The social memoir: an analysis of developing reflective ability in a pre-service methods course» en Teaching and Teacher Education. 20, pp. 59-75.

Carlino, P., (2007) Escribir, leer y aprender en la universidad: una introducción a la alfabetización académica. Buenos Aires: Fondo de Cultura Económica.

Carter, K. y V. Richardson, (1989) «A curriculum for an initial year of teaching program» en The Elementary School Journal. 89 (4), pp.405-419

Concha, S., Aravena, S., Coloma, C. y V. Romero, (2010) «Escritura expositiva en tres niveles de escolaridad: coherencia y dominio de recursos lingüísticos» en Literatura y Lingüística. 21, pp. 75-92.

Concha, S. y J. Paratore, (2011) «Local coherence in persuasive writing: an exploration of chilean students metalinguistic knowledge, writing process and writing products» en Written Communication. 28 (1) pp. 34-69.

Darling-Hammond, L. y J. Bransford, (2005) Preparing teachers for a changing world: What teachers should learn and be able to do. San Francisco, CA: John Wiley and Sons.

Davis, E. (2006) «Characterizing productive reflection among preservice elementary teachers: seeing what matters» en Teaching and Teacher Education. 22 (3), 281-301.

Emig, J. (1977) «Writing as a mode of learning» en College Composition and Communication. 28 (2), pp. 122-128.

Fund, Z., Court, D. y C. Kramarski, (2002) «Construction and application of an evaluative tool to assess reflection in teacher training courses» en Assessment and Evaluation in Higher Education. 27 (6), pp. 485-499.

Harrington, H., Quinn-Leering, K. y L. Hodson, (1996) «Written case analysis and critical reflection» en Teaching and Teacher Education. 12 (1), pp. 25-37.

Herman, W., (1998) «Promoting pedagogical reasoning as preservice teachers analyze case vignettes» en Journal of Teacher Education. 49 (5), pp.391-397.

Kim, H. y J. Hannafin, (2008) «Situated case-based knowledge: An emerging framework for prospective teacher learning» en Teaching and Teacher Education. 24, pp. 1837-1845.

Lee, H. (2005) «Understanding and assessing preservice teachers' reflective thinking» en Teaching and Teacher Education. 21, pp. 699-715. 
LeFevre, F. (2011) «Creating and facilitating a teacher education curriculum using preservice teachers' autobiographical stories» en Teaching and Teacher Education. 38, pp. 890-898.

Luk, J. (2008) «Assessing teacher practicum reflections: Distinguishing discourse features of the "high" and "low" grade reports» en System. 36, pp. 624-641.

Mclellan, E. (2008) «Pedagogical literacy: what it means and what it allows» en Teaching and Teacher Education. 24, pp. 986-1992.

Schemp, P. y S. Woorons, (2006) «Learning to see: developing the perception of an expert teacher» en Journal of physical education, recreation and dance, 77 (6), PP. 29-33.

Schön, D.A. (1983) The reflective practitioner: how professionals think in action. New York: Basic Books.

Silcock, P. (1994) «The process of reflective teaching» en British Journal of Educational Studies, 42 (3), pp. 273-285.

Schleppegrell, M. y L. Oliveira, (2006) «An integrated language and content approach for history teachers» en Journal of English for Academic Purposes. 5, pp. 254-268.

Schleppegrell, M., Greer, S. y S. Taylor, (2008) «Literacy in history: Language and meaning» en Australian Journal of Language and Literacy. 31 (2), pp. 174-187.

Shulman, L. (1987) «Knowledge and Teaching: Foundations of the New Reform» en Harvard Educational Review. 57 (1) Disponible en http:// people.ucsc.edu/ ktellez/shulman.pdf

Snow, C. y P. Uccelli, (2008). «The challenge of academic language» en Olson, D. y Torrance, N.(Eds), The Cambridge Handbook of Literacy. New York: Cambridge University Press, pp. 112-133.

Tapia, M., Burdiles, G. y B. Arancibia, (2003) «Aplicación de una pauta diseñada para evaluar informes académicos universitarios» en Revista Signos 36. (54), pp. 249-257.

Uccelli, P., Dobbs, C. y J. Scott, (en prensa) Mastering academic language: organization and stance in the persuasive writing of high school students.

Youngs, P. y T. Bird, (2010) «Using embedded assessments to promote pedagogical reasoning among secondary teaching candidates» en Teacher and teaching education. 26 (2), pp. 185-198.

Recibido: 28/12/2012

Aceptado: 13/05/2013 